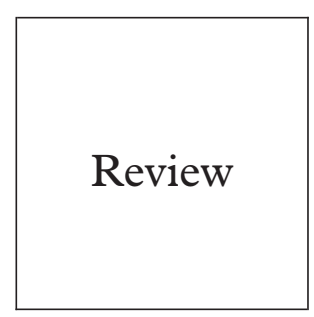

\title{
Anal intraepithelial neoplasia in HIV positive people
}

\author{
Fabiola Martin, Mark Bower
}

Objective: To review the current literature on HIV associated anal intraepithelial neoplasia (AIN).

Methods: A comprehensive Medline/Pubmed search was performed for the years 1980-2001 (January) for articles pertaining to HIV associated anal intraepithelial neoplasia. From the MeSH terms "anal intraepithelial neoplasia" and "anal cancer" the following subheadings were used: HIV, homosexual men, HPV, Epidemiology, Etiology, Mortality, Diagnosis, Screening, Drug Therapy, Surgical Therapy, Radio Therapy, Risk factors, ASIL. The search was limited to "human" for all searches. In the absence of enough "randomised controlled trials" the search was extended to clinical trials, reviews, and case reports. One analysis on cost effectiveness and two abstracts presented at 12th World AIDS Conference and 6th Conference on Retrovirus and Opportunistic Infections were included. The 44 publications referred to originate from the United Kingdom (9), the United States (26), and Denmark (5), with one each from Switzerland, Germany, Australia, and France. The Cochrane Database of systematic reviews yielded 11 complete reviews for "anal cancer" and none for "anal intraepithelial neoplasia." The textbook of AIDS-related cancers and their treatment was consulted. We also included our personal experience from the treatment of patients at the Chelsea and Westminster Hospital, one of the largest centres for the management of HIV disease in Europe.

Conclusion: Routine anal cytological screening followed by appropriate management of AIN is an important issue for HIV infected patients. The natural history of AIN has not been fully established and this prevents clinicians from defining clear management protocols. There is early evidence that the benefits of highly active antiretroviral therapy (HAART) in terms of restoring immune function and reducing opportunistic infections and some neoplasms may not extend to regression of AIN. Under these circumstances it might be predicted that AIN and subsequent progression to invasive anal cancer would rise as HAART prolongs the lives of seropositive people. However, routine anal cytological screening will surely have to await an effective proved intervention for AIN and this would seem to be a pressing clinical goal.

(Sex Transm Inf 2001;77:327-331)

Keywords: anal intraepithelial neoplasia; HIV; human papillomavirus

\section{Introduction}

Squamous tumours of the anogenital tract share histological, epidemiological, and pathogenetic similarities. ${ }^{1}$ Moreover, both anal cancer and cervical cancer are thought to arise from the progression of squamous intraepithelial lesions to invasive tumours. ${ }^{2}$ Anal intraepithelial neoplasia (AIN) was originally described by Fenger and Nielsen who reviewed all anal surgical pathological specimens in Denmark over a 2 year period. ${ }^{3}$ They graded AIN using the same system in use for cervical intraepithelial neoplasia (CIN), defining AIN grade 1 as nuclear abnormalities confined to the lower third of the epithelium, AIN grade 2 to the lower two thirds, and in AIN grade 3 nuclear abnormalities are present throughout the full thickness.

Department of

Oncology, Chelsea and

Westminster Hospital,

Fulham Road, London

SW10 9NH, UK

F Martin

Mark Bower

Correspondence to:

Mark Bower

m.bower@ic.ac.uk

Accepted for publication 17 August 2001

\section{Epidemiology}

The incidence of anal cancer in the general population is very low. Every year 300 (0.5 per $100000)$ new cases are diagnosed in the United Kingdom and it only accounts for $1.5 \%$ of digestive system cancers in the United States. ${ }^{5}$ However, men who have sex with men were found to have a high incidence of invasive anal cancer even before the emergence of the HIV pandemic. ${ }^{67}$ The incidence of anal cancer among homosexual men in the pre-AIDS era was estimated to be $35 / 100000,{ }^{8}$ which resembles the incidence of cervical cancer before the introduction of routine cervical smear screening. The risk of anal cancer is even higher among people with HIV infection, ${ }^{9}$ although unlike invasive cervical cancer, invasive anal cancer is not an AIDS defining diagnosis. A recent analysis of a register of 309000 US patients with HIV infection found that the relative risk of invasive anal cancer in men with HIV infection is 37.9 and for women with HIV is $6.8 .^{10}$ Nevertheless, meta-analysis of 23 prospective cohort studies including 47936 HIV seropositive people included only 18 patients with invasive anal cancer and changes in incidence in the era of highly active antiretroviral therapy (HAART) could not be estimated. ${ }^{11}$ The risk of precursor lesions including in situ cancers was also estimated from the registry data. The relative risk of in situ anal cancers in HIV seropositive people is even higher with a value of 60 in men and 7.8 for women. Indeed, the relative risk for in situ anal cancer in women exceeds that for cervical or vulval/vaginal in situ cancer. ${ }^{10}$ Sayers et al measured the prevalence in Edinburgh of anal cytological abnormalities in groups of HIV positive and 
negative homosexual men and compared them with those of heterosexual men. AIN was seen in $30 \%$ of satisfactory anal smears from HIV positive men, in $4.7 \%$ of HIV negative men, and in none of the heterosexual men. ${ }^{12}$

These findings support the earlier results that investigated the epidemiology of anal squamous intraepithelial lesions (ASIL). A prospective cohort of $158 \mathrm{HIV}$ positive and 147 HIV negative homosexual men was studied for a mean period of 20 months. Among men with negative anal cytology and proctoscopy at enrolment, $15 \%$ of HIV positive and $5 \%$ of HIV negative men developed high grade ASIL (HSIL). This study also defined a relative risk of 7.5 for developing HSIL in HIV positive homosexual men if the CD4 count was below $500 \times 10^{6} / 1 .{ }^{13}$ In 1998 Palefsky and his group at the University of California, San Francisco, observed the natural history of ASIL in a cohort study of $346 \mathrm{HIV}$ positive and 262 HIV negative homosexual men over a 2 year period. ${ }^{14}$ HSIL developed in $20 \%$ of HIV positive and $8 \%$ of HIV negative men who had normal baseline evaluations, while $62 \%$ of HIV positive and $36 \%$ of HIV negative subjects who had low grade ASIL (LSIL) at enrolment developed HSIL.

\section{Histopathology}

In the anal canal the transformation zone, which separates the columnar epithelium of the rectum from the keratinising anal squamous mucosa, lies above the dentate line and is the site where most anal intraepithelial neoplasms arise. ${ }^{15} \mathrm{~A}$ series of histopathological changes occur in this transformation zone, which range from atypical squamous cells of undetermined significance (ASCUS) to condylomata to anal squamous intraepithelial lesions (ASIL); they are thought, at least in part, to be manifestations of infection with human papillomavirus (HPV). ASIL may be divided into low grade anal squamous intraepithelial lesions (LSIL) and high grade anal squamous intraepithelial lesions (HSIL). In LSIL, which is equivalent to anal intraepithelial neoplasia grade 1 (AIN 1), less than a third of the epithelial layer is replaced with immature basaloid cells, while up to two thirds are replaced in AIN 2 and more than two thirds in AIN 3. An additional feature of HSIL is microinvasion through the basement membrane. Epidemiological studies support the theory that anal cancers arise from a stepwise progression from subclinical infection with high risk HPV, through LSIL and HSIL to invasive anal cancer. Each step is thought to be accompanied by molecular and immunological changes analogous to the pathogenetic process described for colon cancer. ${ }^{16}$

\section{Molecular virology}

Epidemiological data suggest that the development of ASIL and progression from LSIL to HSIL are closely related to infection with high risk genotypes of HPV. ${ }^{17-19}$ In addition, the prevalence of ASIL rises with an increasing number of HPV genotypes detected by polymerase chain reaction (PCR). ${ }^{20}$ In addition, the prevalence of HPV infection is higher in HIV positive men than in HIV negative men and particularly in men with CD 4 counts $<200$ $\times 10^{6} / 1^{13}{ }^{18} 21-23$ Despite these links it remains uncertain whether the high incidence of HPV associated malignancies in people with HIV infection is related to increased exposure to HPV, a high prevalence of other co-factors, or HIV related immunosuppression.

Human papillomaviruses are DNA papovaviruses that have an $8 \mathrm{~kb}$ genome. Over 70 genotypes of HPV have been identified and many are tropic for squamous epithelium. These DNA viruses may cause non-replicative infection in some cell types as part of their normal life cycle. The viral DNA persists in host cells as episomes. HPV expresses latent phase genes that can enable the virus to persist or to increase the number of latently infected cells. These early region genes control viral DNA replication, transcription regulation, and cellular transformation, while late region genes encode the structural viral capsid proteins. Certain HPV genotypes are particularly associated with anogenital infection, dysplasia, and malignancy including HPV genotypes 16, 18, 31,33 , and 35 . These genotypes cause cellular proliferation through two early region genes expressed during latent infection, E6 and E7. E6 binds directly to the tumour suppressor protein p53 and to another cell protein, E6 associated protein (E6AP). The binding of E6 to E6AP and to p53 results in the degradation of p53 via the ubiquitin pathway. In contrast, E7 binds directly to the retinoblastoma (Rb) tumour suppressor protein $\mathrm{p} 105 \mathrm{Rb}$ and to the p107 and p130 Rb related proteins, releasing $\mathrm{E} 2 \mathrm{~F}$ related transcription factors from $\mathrm{Rb}$. The liberated E2F promotes transcription of several genes essential for cell and viral DNA replication and for the progression of cells into and through G1 and into the $S$ phase of the cell cycle. In addition, E7 may alter the interactions between $\mathrm{Rb}$ and cyclins and other cell cycle regulatory proteins. Thus, HPV targets both of the major guardians of cellular proliferation, p53 and Rb. The expression of E6 and E7 genes by high risk HPV types in epithelial cells appears to be adequate for cellular immortalisation, but not for full cell transformation, as measured by the ability to proliferate in nude or severe combined immunodeficiency (SCID) mice. These observations are consistent with the clinical evidence that the development of a fully malignant phenotype occurs long after infection by HPV within a subpopulation of chronically infected cells, which presumably accumulate further mutations. ${ }^{24}$

\section{Risk factors}

Established risk factors for the development of ASIL include HIV seropositivity, low CD4 cell count, persistent HPV infection, high risk HPV genotypes, and infection with multiple HPV genotypes. No association between ASIL and other sexually transmitted diseases such as herpes, gonorrhoea, or syphilis has been found. ${ }^{18}{ }^{25}$ Other independent risk factors for ASIL are a history of anal intercourse and smoking. ${ }^{19}$ Smoking at least one pack of cigarettes per day is thought to affect both the 
anal mucosa directly through circulating carcinogens and indirectly through suppression of cell mediated immunity. ${ }^{18}$ ASIL is also associated with iatrogenic immunosuppression in patients who have received an organ allograft. ${ }^{26}$

In addition, cohort studies have identified factors that predict the progression of low grade LSIL to high grade HSIL and these include HIV status and infection with multiple genotypes of HPV. In a prospective 2 year follow up study in San Francisco, Palefsky et al showed that $70 \%$ of HIV positive and $31 \%$ of HIV negative homosexual men developed ASIL when diagnosed to have ASCUS on anal cytology at base line. In the same study group $62 \%$ of HIV positive men with anal LSIL at baseline progressed to HSIL within 2 years. ${ }^{14}$ Similarly, in a smaller study that enrolled 57 HIV positive homosexual men the prevalence of high grade AIN rose from $10 \%$ at enrolment to $70 \%$ over an average follow up of 17 months. ${ }^{27}$ There are, however, no published data on the rate of regression of LSIL to ASCUS or normal mucosa or of HSIL to LSIL, ASCUS, or normal mucosa.

\section{Clinical features}

ASIL is usually asymptomatic although occasionally patients complain of pain, pruritis, bleeding, discharge, or tenesmus. Indeed, high grade AIN may be detectable despite normal anoscopic mucosal appearance. ${ }^{28}$ Multifocal intraepithelial neoplasia is frequently a field effect and women with AIN should also be examined for cervical, vulval, and vaginal intraepithelial neoplasia (CIN, VIN, and VAIN). ${ }^{2}$ Indeed, a recent study of the prevalence of HPV in women found that anal HPV was commoner than cervical HPV in both HIV seropositive and high risk seronegative women. ${ }^{29}$

\section{Management}

The epidemiology of AIN in the immunocompetent population suggested that the majority of people with AIN probably do not progress to invasive anal cancer. This led to the development of management guidelines for AIN. ${ }^{30}{ }^{31}$ Immunocompetent patients with AIN 1 or AIN 2, after examination for associated intraepithelial neoplasia in women, should be reviewed at 6 monthly intervals and if there is no evidence of cytological progression on three consecutive occasions they may be discharged. Patients with AIN 3 should be treated by surgical excision, ${ }^{32}$ checked after 2 months, and rebiopsied if healing is incomplete. Follow up anoscopy and cytology are advocated every 6 months and the reported recurrence rate is $30 \%$ at 5 years. ${ }^{33}$ Multifocal or extensive AIN 3 may require a wide excision ${ }^{34}$ with skin grafting and even a temporary stoma. These protocols, which arose by an iterative process in one unit, have not been widely implemented.

Moreover, the guidelines may be inadequate for AIN in the context of HIV infection and the clinical management of HIV associated AIN remains uncertain. The higher risk of progression from LSIL to HSIL in HIV seropositive patients suggests that a rather more prolonged surveillance programme is warranted for LSIL and indefinite 6 monthly assessments are advocated. Again active therapy for HSIL has been proposed with excision of larger lesions, although small lesions $\left(<1 \mathrm{~cm}^{2}\right)$ may be treated by topical application of bichloroacetic acid or trichloroacetic acid. Extensive HSIL may require multiple operations and is associated with a risk of residual disease, anal stenosis, and faecal incontinence. ${ }^{35}$

Initial data from cohort studies at UCSF suggest that most anal HSIL lesions do not regress after the initiation of HAART, despite the established benefit of HAART on other viral infections and associated diseases in HIV infected patients. ${ }^{36}$ In contrast, the introduction of HAART has been reported to cause regression of cervical intraepithelial neoplasia, ${ }^{37}$ although there was no reduction of HPV DNA levels in the cervix and this was not a universal finding. ${ }^{38} 39$

\section{Screening}

The high prevalence and progressive behaviour of AIN in the immunocompromised person has led to pilot studies of screening programmes. Analogous to cervical cancer screening, these programmes have used anal cytological screening in high risk populations. The screening for ASIL consists of a swab for anal cytology and anoscopy with 3\% acetic acid and biopsy of visible lesions. Palefsky and colleagues looked at sensitivity, specificity, positive and negative predictive value of anal cytology. ${ }^{40}$ Not surprisingly, including ASCUS in the abnormal category raised the sensitivity and lowered the specificity of anal cytology. Anal cytology had a $69 \%$ sensitivity among HIV positive men and $47 \%$ among HIV negative men at their first visit once ASCUS was included as an abnormal cytology. Its sensitivity was $81 \%$ for the former and $50 \%$ for the latter group for all subsequent visits combined. In comparison, the sensitivity of cervical cytology is $80 \%$ using colposcopy and histopathology as a gold standard. The specificity of anal cytology was $59 \%$ in HIV positive men and $92 \%$ in HIV negative men when including ASCUS. Cost effectiveness modelling has also been performed to evaluate anal cytology screening in HIV positive men with CD4 cell counts under $500 \times 10^{6} / 1$ based on the assumption that HSIL would progress to anal cancer within 3 years. It was concluded that screening HIV positive homosexual men annually and HIV negative homosexual men every $2-3$ years for HPV induced anal disease would be cost effective and would prolong life expectancy. ${ }^{41} 42$

\section{Future}

Routine anal cytological screening followed by appropriate management of AIN is an important issue for HIV infected patients. There is early evidence that the benefits of HAART in terms of restoring immune function and reducing opportunistic infections and some neoplasms may not extend to regression of AIN. Under these circumstances it might be predicted that AIN and subsequent progression 
to invasive malignancy would rise as HAART prolongs the lives of seropositive people.

A review of the tumour registry from 1980 to 1999 at the University of Texas Southwestern Medical Centre in Dallas identified 73 patients with anal squamous carcinoma, including 23 who were HIV positive. In addition, nine patients had in situ carcinomas treated by local excision. Patients with in situ carcinomas were younger, supporting the hypothesis of progression from in situ to invasive disease. Among patients with invasive disease a low CD4 cell count predicted a worse survival while taking HAART was associated with a favourable prognosis. Most of these patients succumbed to opportunistic infections rather than anal cancer and the authors use this evidence to support the case for invasive anal cancer being included as an AIDS defining diagnosis. Finally, the authors note that chemoradiotherapy was better tolerated in patients receiving concomitant HAART. ${ }^{43}$ Whether in situ disease should be an indication for starting HAART therapy remains unclear since there is little evidence of regression of AIN with HAART.

Many publications support the use of combination chemoradiotherapy based on 5-fluorouracil and mitomycin for treatment of invasive anal carcinoma. ${ }^{44}$ This is standard practice in HIV patients in our unit. ${ }^{46}$ In addition to the surgical treatments described earlier, several pharmacological approaches have been advocated for the management of in situ disease, although few have been evaluated formally. Oral isotretinoin, systemic interferon $\alpha$, topical 5-fluorouracil, podophyllum, and imiquimod have all been used here and elsewhere. ${ }^{47}$ A recent case report reports the successful management of HIV associated in situ anal squamous cell carcinoma with a 16 week course of $5 \%$ imiquimod cream three times weekly and daily $5 \%$ fluorouracil cream. ${ }^{48}$

Although in the absence of a clear management strategy a national screening policy for HIV positive homosexual men cannot be advocated, pilot programmes should be established offering anal cytology screening programmes at centres with high attendance of HIV positive homosexual men. The aim of these pilots should be to establish further long term prospective data on the progression and prognosis of AIN and identify patients who are eligible for therapeutic intervention studies.

Contributors: FM performed the literature search and prepared the initial draft of the manuscript; MB performed an additional literature search, wrote the section on personal experience at Chelsea and Westminster Hospital, and finalised the manuscript.

1 Melbye M, Sprogel P. Aetiological parallels between anal cancer and cervical cancer. Lancet 1991;338:657-9.

2 Scholefield J, Sonnex C, Talbot I, et al. Anal and cervical intraepithelial neoplasia: possible parallel. Lancet 1989;2: $765-9$.

3 Fenger C, Nielsen V. Dysplastic changes in the anal canal epithelium in minor surgical specimens. Acta Pathol Microbiol Immunol Scand 1981;89:463-5.

4 Fenger C, Nielsen V. Intraepithelial neoplasia in the anal canal. Acta Pathol Microbiol Immunol Scand 1986;94:343-9.

5 Greenlee RT, Murray T, Bolden S, et al. Cancer statistics. Greenlee RT, Murray T, Bolden

6 Daling J, Weiss N, Klopfenstein L, et al. Correlates of homosexual behaviour and the incidence of anal cancer. homosexual behaviour and
7 Wexner S, Milsom J, Dailey T. The demographics of anal cancer are changing. Dis Col Rect 1987;30:942-6.

8 Daling J, Weiss N, Hislop T, et al. Sexual practices, sexually transmitted diseases, and the incidence of anal cancer. $N$ Engl f Med 1987;317:973-7.

9 Melbye M, Cote TR, Kessler L, et al. High incidence of anal cancer among AIDS patients. Lancet 1994;343:636-9.

10 Frisch M, Biggar R, Goedert J, et al. Human papillomavirusassociated cancers in patients with human immunodeficiency virus infection and acquired immunodeficiency syndrome. F Natl Cancer Inst 2000;92:1500-10.

11 International Collaboration on HIV and Cancer. Highly active antiretroviral therapy and incidence of cancer in active antiretroviral therapy and incidence of cancer in
human immunodeficiency virus-infected adults. $\mathcal{F}$ Natl haman immunodeficiency $2000 ; 92: 1823-30$.

12 Sayers S, McMillan A, McGoogan E. Anal cytological abnormalities in HIV-infected homosexual men. Int $\mathcal{F}$ STD AIDS 1998;9:37-40.

13 Critchlow C, Surawicz C, Holmes K, et al. Prospective study of high-grade anal squamous intraepithelial neoplasia in a cohort of homosexual men: influence of HIV infection, immunosuppression and human papillomavirus infection. AIDS 1995;9:1255-62.

14 Palefsky J, Holly E, Hogeboom C, et al. Virologic, immunologic, and clinical parameters in the incidence and progression of anal squamous intra-epithelial lesions in HIVpositive and HIV-negative homosexual men. If Aquir positive and HIV-negative homosexual men. F

15 Palefsky J. Anal human papillomavirus infection and anal cancer in HIV-positive individuals: an emerging problem. AIDS 1994;8:283-95.

16 Fearon ER, Vogelstein B. A genetic model for colorectal tumorigenesis. Cell 1990;61:759-67.

17 Palefsky J. Anal squamous intraepithelial lesions: relation of HIV and human papillomavirus infection. If AIDS 1991;21(suppl):S42-8.

18 Palefsky J, Shiboski S, Moss A. Risk factors for anal human papillomavirus infection and anal cytological abnormalities in HIV-negative homosexual men. $f$ Acquir Immune Defic Syndr 1994;7:599-606.

19 Palefsky J, Holly E, Ralston M, et al. Anal squamous intraepithelial lesions in HIV-positive and HIV-negative homosexual and bisexual men:prevalence and risk factors. f Acquir Immune Defic Syndr Hum Retrovirol 1998;17:3206.

20 Friedman HB, Saah AJ, Sherman M, et al. Human papillomavirus, anal squamous intraepithelial lesions, and human mmunodeficiency virus in a cohort of gay men. F Infect Dis 1998;178:45-52.

21 Caussey D, Goedert J, Palefsky J, et al. Interaction of human immunodeficiency and papilloma viruses: association with anal epithelial abnormality in homosexual men. Int $\mathcal{f}$ Cancer 1990;46:214-19.

22 Melbye M, Palefsky J, Gonzales J, et al. Immune status as a determinant of human papillomavirus infection and its correlation with anal epithelial abnormalities. Int 7 Cancer 990;46:203-6.

23 Palefsky JM, Holly EA, Ralston M, et al. High incidence of anal high-grade squamous intra-epithelial lesions among HIV-positive and HIV-negative homosexual and bisexual men. AIDS 1998;12:495-503.

24 Zur Hausen H. Immortalisation of human cells and their malignant conversion by high risk human papillomavirus genotypes. Cancer Biol 1999;9:405-11.

25 Law C, Qassim M, Thompson C, et al. Factors associated with clinical and sub-clinical anal human papillomavirus infection in homosexual men. Genitourin Med 1991;67:92infecti

26 Ogunbiyi O, Scholefield J, Raferty A, et al. Prevalence of anal human papillomavirus infection and anal intraepithelial neoplasia in renal allograft recipients. $\mathrm{Br} F \mathrm{f}$ Surg 1994;81:365-7.

27 Lacey H, Wilson G, Tilston P, et al. A study of anal intraepithelial neoplasia in HIV positive homosexual men. Sex Transm Inf 1999;75:172-7.

28 Surawicz C, Critchlow C, Sayer J, et al. High grade anal dysplasia in visually normal mucosa in homosexual men: seven cases. Am f Gastroenterol 1995;90:1776-8.

29 Palefsky J, Holly E, Ralston M, et al. Prevalence and risk factors for anal human papillomavirus infection in human immunodeficiency virus (HIV)-positive and high risk HIVnegative women. F Infect Dis 2001;183:383-91.

30 Scholefield J, Johnson J, Hitchcock A, et al. Guidelines for anal cytology-to make cytological diagnosis and follow up anal cytology - to make cytological diagnosis and

31 Scholefield J. Anal intraepithelial neoplasia. Br f Surg 1999; 86:1363-4.

32 Cleary R, Schaldenbrand J, Fowler J, et al. Perianal Bowen's disease and anal intraepithelial neoplasia: review of the literature. Dis Colon Rectum 1999;42:945-51.

33 Marchesa P, Fazio V, Oliart S, et al. Perianal Bowen's disease - a clinicopathologic study of 47 patients. Dis Colon Rectum 1997;40:1286-93.

34 Lyons M, Francis N, Allen-Mersh T. Treatment of grade 3 anal intraepithelial neoplasia by complete anal mucosa excision without faecal diversion: report of a case. Dis Colon Rectum 1999;42:1342-4

35 Brown S, Skinner P, Tidy J, et al. Outcome after surgical resection for high grade anal intraepithelial neoplasia (Bowen's disease). Br ₹ Surg 1999;86:1063-6.

36 Palefsky JM, Holly EA, Ralston ML, et al. The effect of HAART on the natural history of anal squamous intraepithelial lesion in HIV+ men. $\mathcal{F}$ AIDS 1999;21:A13. 
37 Heard I, Schmitz V, Costagloila D, et al. Early regression of cervical lesions in HIV seropositive women receiving highly active antiretroviral treatment (HAART). AIDS 1998;12: 1459-64.

38 Hoesly C, Jin G, Bragg S, et al. Molecular epidemiology of $\mathrm{HPV}$ infection in the genital tract of HIV seropositive women. 6th Conference on Retroviruses and Opportunistic Infections. Chicago, 1999: Abstract 465.

39 Sperandeo D, Robaglia-Schlupp A, Cau P, et al. Trying to know the role of viral load and ARV therapies in the progression of cervical disease. 12th World AIDS Conference. Geneva, 1998: Abstract 22321.

40 Palefsky J, Holly E, Hogeboom C, et al. Anal cytology as a screening tool for anal squamous intraepithelial lesions. $f$ Acquir Immune Defic Syndr Hum Retrovirol. 1997;14:415-22.

41 Goldie SJ, Kuntz KM, Weinstein MC et al. The clinical effectiveness and cost-effectiveness of screening for anal squamous intraepithelial lesions in homosexual and bisexual HIV-positive men. $¥ A M A$ 1999;281:1822-9.

42 Goldie SJ, Kuntz KM, Weinstein MC, et al. Costeffectiveness of screening for anal squamous intraepithelial lesions. Am ₹ Med 2000;108:634-41.
43 Place R, Gregorcyk S, Huber P, et al. Outcome analysis of HIV-positive patients with anal squamous cell carcinoma. Dis Colon Rectum 2001;44:506-12.

44 Bieri S, Allal A, Kurtz J. Sphincter-conserving treatment of carcinoma of the anal margin. Acta Oncol 2001;40:20-33.

45 UKCCCR Anal Cancer Trail Working Party. UK Coordinating Committee on Cancer Resaerch. Epidermoid anal cancer: results from the UKCCCR randomised trail of adiotherapy alone versus radiotherapy, 5 -fluorouraci, and mitomysin. Lancet 1996;348:1049-54.

46 Cleator S, Fife $\mathrm{K}$, Nelson $\mathrm{M}$, et al. Treatment of HIV-associated invasive anal cancer with combined chemoradiation. Eu f Cancer 2000;36:754-8.

47 Palefsky J. Anal squamous intraepithelial lesions in HIVpositive men and women. In: Feigal EG, Levine AM, Biggar RJ, eds. AIDS-related cancers and their treatment. New York: Marcel Dekker, 2000.

48 Pehoushek M, Smith K. Immiquimod and 5\% fluorouracil therapy for anal and perineal squamous cell carcinoma in situ in an HIV-1 positive man. Arch Dermatol 2001;137:14-

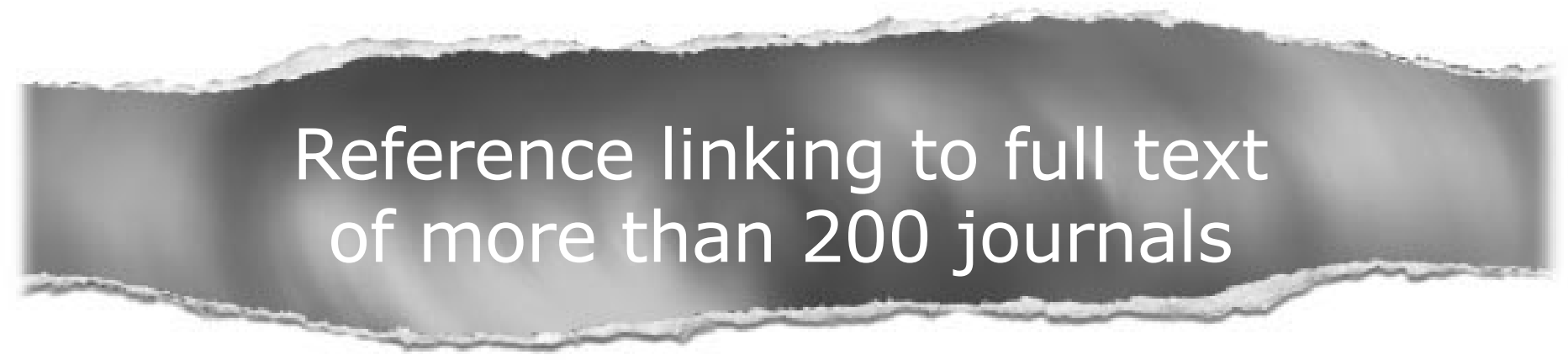

Toll free links

You can access the FULL TEXT of articles cited in Sexually Transmitted Infections online if the citation is to one of the more than 200 journals hosted by HighWire (http://highwire.stanford.edu) without a subscription to that journal. There are also direct links from references to the Medline abstract for other titles.

www.sextransinf.com 